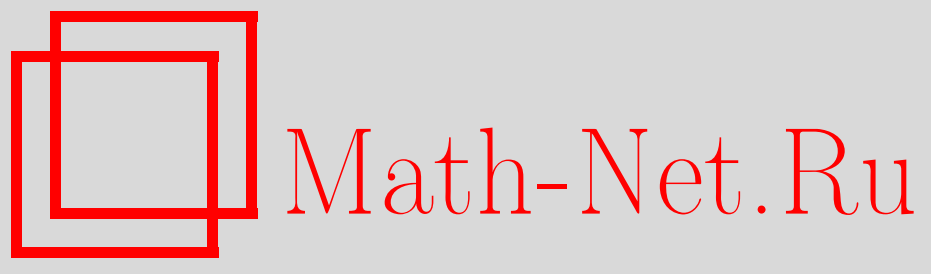

В. А. Ватутин, Предельная теорема для промежуточно докритического ветвящегося процесса в случайной среде, Теория вероятн. и ее примен., 2003, том 48, выпуск 3, 453-465

DOI: https://doi.org/10.4213/tvp265

Использование Общероссийского математического портала MathNet.Ru подразумевает, что вы прочитали и согласны с пользовательским соглашением

http://www . mathnet.ru/rus/agreement

Параметры загрузки:

IP : 34.229 .108 .108

26 апреля 2023 г., 13:50:36

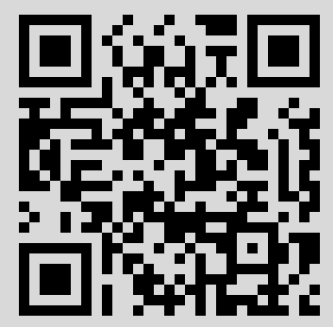




\section{ПРЕДЕЛЬНАЯ ТЕОРЕМА ДЛЯ ПРОМЕЖУТОЧНО ДОКРИТИЧЕСКОГО ВЕТВЯЩЕГОСЯ ПРОЦЕССА В СЛУЧАЙНОЙ СРЕДЕ}

Найдена асимптотика вероятности невырождения промежуточно докритического ветвящегося процесса $Z_{n}$ в случайной среде в случае, когда некоторое преобразование закона распределения числа непосредственных потомков частиц принадлежит области притяжения устойчивого закона с параметром $\alpha \in(1,2]$. Доказано, что распределение случайной величины $\left\{Z_{n}\right\}$ при условии $Z_{n}>0$ сходится к невырожденному распределению при $n \rightarrow \infty$.

Ключевые слова $и$ фразы: ветвяшиеся процессы в случайной среде, вероятность невырождения, промежуточно докритический процесс, предельная теорема, случайные блуждания, устойчивые распределения.

1. Введение и основной результат. Ветвяшиеся процессы в случайной среде (ВПСС) являются одним из интереснейших обобщений ветвящихся процессов Гальтона-Ватсона. В этой статье мы рассматриваем простейший случай таких процессов, а именно, ситуацию, когда последовательные состояния среды независимы и одинаково распределены. Модель, которая нас интересует, описывается следующим образом. Пусть последовательные состояния сред задаются бесконечномерными векторами $\left\{\pi_{n}, n \in \mathbf{N}_{0}\right\}$, где

$$
\begin{aligned}
& \pi_{n}=\left\{\pi_{n}^{(0)}, \pi_{n}^{(1)}, \pi_{n}^{(2)}, \ldots\right\}, \quad \pi_{n}^{(i)} \geqslant 0, \\
& \sum_{i=0}^{\infty} \pi_{n}^{(i)}=1, \quad n \in \mathbf{N}_{0}=\{0,1,2, \ldots\},
\end{aligned}
$$

причем наборы $\pi_{n}$ независимы и одинаково распределены. С вектором $\pi_{n}$ мы свяжем производящую функцию

$$
f_{n}(s)=\sum_{i=0}^{\infty} \pi_{n}^{(i)} s^{i} .
$$

* Математический институт им. В. А. Стеклова РАН, ул. Губкина, 8, 119991 Москва, ГСП-1, Россия; e-mail: vatutin@mi.ras.ru

1) Работа выполнена при финансовой поддержке РФФИ (грант 02-01-00266), РФФИ-ННИО (грант 02-01-04002) и гранта НШ-1758.2003.1 Президента Российской Федерации для поддержки ведуших научных школ. 
Для удобства введем также вероятностную производящую функцию $f(s) \stackrel{d}{=} f_{n}(s)$, не зависяцую от набора $f_{n}(s), n=0,1,2, \ldots$ В этих терминах эволюция интересующего нас ветвящегося процесса в случайной среде $\left\{Z_{n}, n \in \mathbf{N}_{0}\right\}$ описывается соотношениями

$$
Z_{0}=1, \quad \mathbf{E}\left[s^{Z_{n+1}} \mid f_{0}, f_{1}, \ldots, f_{n} ; Z_{0}, Z_{1}, \ldots, Z_{n}\right]=\left(f_{n}(s)\right)^{Z_{n}} .
$$

Пусть $a=\mathbf{E} \ln f^{\prime}(1)$. ВПСС называется надкритическим, если $a>0$, критическим, если $a=0$, и докритическим, если $a<0$. В данной работе мы рассматриваем докритические ВПСС. Такие процессы исследовались многими авторами (см., например, [1], [2], [3], [5], [7], [8], [13], [16]).

Как оказалось, свойства докритических ВПСС существенно зависят от значения параметра $b=\mathbf{E} f^{\prime}(1) \ln f^{\prime}(1)$.

Докритический ВПСС называется строго докритическим, если $b<0$, промежуточно докритическим, если $b=0$, и слабо докритическим, если $b>0$. Поскольку докритические ВПСС вырождаются с вероятностью единица, естественно исследовать поведение вероятности невырождения $\mathbf{P}\left\{Z_{n}>0\right\}$ при $n \rightarrow \infty$. Асимптотика вероятности невырождения докритических процессов всех типов для случая, когда производящие функции числа потомков дробно-линейны, была найдена В. И. Афанасьевым (см., например, [1], [2]). Обший случай рассматривался в [7] и [8]. В работе [8] было показано, что для строго докритического процесса общего вида, удовлетворяющего условию $\mathbf{E} Z_{1} \ln ^{+} Z_{1}<\infty$, существует константа $d \in(0, \infty)$ такая, что

$$
\mathbf{P}\left\{Z_{n}>0\right\} \sim d\left(\mathbf{E} Z_{1}\right)^{n}, \quad n \rightarrow \infty .
$$

Обозначим $I\{A\}$ индикатор события $A$. В [7] установлено, что для промежуточно докритического ВПСС общего вида, удовлетворяющего условиям

$$
\begin{gathered}
\mathbf{E}\left[f^{\prime}(1)\left(\ln f^{\prime}(1)\right)^{2}\right]<\infty, \\
\mathbf{E}\left[f^{\prime \prime}(1)\left(1-\ln f^{\prime}(1) I\left\{f^{\prime}(1)<1\right\}\right)\right]<\infty,
\end{gathered}
$$

существует константа $d \in(0, \infty)$ такая, что

$$
\mathbf{P}\left\{Z_{n}>0\right\} \sim d n^{-1 / 2}\left(\mathbf{E} Z_{1}\right)^{n}, \quad n \rightarrow \infty,
$$

в то время как для слабо докритического ВПСС с параметром $\kappa$, определяемым равенством

и

$$
\mathbf{E}\left[\left(f^{\prime}(1)\right)^{\kappa}\right]=\min _{0 \leqslant \theta \leqslant 1} \mathbf{E}\left[\left(f^{\prime}(1)\right)^{\theta}\right]
$$

$$
\mathbf{E} f^{\prime}(1) \ln f^{\prime}(1)<\infty, \quad \mathbf{E}\left[\frac{f^{\prime \prime}(1)}{f^{\prime}(1)^{1-\kappa}}+\frac{f^{\prime \prime}(1)}{f^{\prime}(1)^{2-\kappa}}\right]<\infty,
$$

существует константа $d \in(0, \infty)$ такая, что

$$
\mathbf{P}\left\{Z_{n}>0\right\} \sim d n^{-3 / 2}\left(\mathbf{E}\left[\left(f^{\prime}(1)\right)^{\kappa}\right]\right)^{n}, \quad n \rightarrow \infty .
$$


Цель данной заметки - найти асимптотику вероятности невырождения промежуточно докритических ВПСС при более слабых, чем (2)-(3), ограничения $\mathrm{x}$ и доказать условную предельную теорему о распределении числа частиц в процессе в момент $n$ при условии невырождения процесса к этому моменту.

Пусть

$$
X:=\ln f^{\prime}(1), \quad \eta:=f^{\prime \prime}(1)\left(f^{\prime}(1)\right)^{-2} .
$$

Будем говорить, что выполнено условие $\mathscr{A}$, если

(A1) распределение случайной величины $X$ абсолютно непрерывно и либо выполнено условие (2), либо условие (2) нарушено, но существуют $\alpha \in(1,2]$ и медленно меняющаяся на бесконечности функция $L(t)$ такие, что

$$
\mathbf{E} f^{\prime}(1) I\left\{\ln f^{\prime}(1)>t\right\}=\mathbf{E} e^{X} I\{X>t\} \sim \frac{L(t)}{t^{\alpha}}, \quad t \rightarrow \infty ;
$$

(A2) для $\alpha<2$ найдутся $\varepsilon_{0} \in(0,1]$ и $\delta_{0} \in(0,1)$ такие, что $\mathbf{E}\left[e^{X} \eta^{\varepsilon_{0}}\left((1+X)^{\alpha-1+\delta_{0}} I\{X \geqslant 0\}+(1-X)^{\alpha-1+\delta_{0}} e^{\varepsilon_{0} X} I\{X<0\}\right)\right]<\infty$;

(A3) при $\alpha=2$ найдется $\varepsilon_{0} \in(0,1)$ такое, что соотношение $(6)$ справедливо с $\delta_{0}=0$.

Отметим, что аналог условия (5) возникает естественным образом при исследовании вероятностей больших уклонений крамеровского типа в случае, когда сопряженное распределение имеет «тяжелый» хвост (см., например, [12]).

Теорема 1. Пусть $Z_{n}$ - промежуточно докритический ВПСС, удовлетворяюший условию $\mathscr{A}$. Тогда существует медленно меняющаяся на бесконечности функиия $L_{1}(n)$, такая, что

$$
\mathbf{P}\left\{Z_{n}>0\right\} \sim n^{-1 / \alpha} L_{1}(n)\left(\mathbf{E} Z_{1}\right)^{n}, \quad n \rightarrow \infty .
$$

Кроме того, для любого $s \in[0,1)$

$$
\lim _{n \rightarrow \infty} \mathbf{E}\left[s^{Z_{n}} \mid Z_{n}>0\right]=\phi(s),
$$

где $\phi(s)$ - производящая функиия собственной случайной величины.

3 а м е ч а н и е 1 . При $\alpha=2$ и $\varepsilon_{0}=1$ условие (6) принимает вид $\mathbf{E}\left[\frac{f^{\prime \prime}(1)}{f^{\prime}(1)}\left(1+\ln f^{\prime}(1)\right) I\left\{f^{\prime}(1) \geqslant 1\right\}+f^{\prime \prime}(1)\left(1-\ln f^{\prime}(1)\right)\left\{f^{\prime}(1)<1\right\}\right]<\infty$.

Поскольку величина $\left(f^{\prime}(1)\right)^{-1} \ln f^{\prime}(1)$ ограничена при $f^{\prime}(1) \geqslant 1$, то найдется константа $c>0$ такая, что

$$
\begin{aligned}
\frac{f^{\prime \prime}(1)}{f^{\prime}(1)}\left(1+\ln f^{\prime}(1)\right) I\left\{f^{\prime}(1) \geqslant 1\right\} & \leqslant f^{\prime \prime}(1)\left(1+\frac{\ln f^{\prime}(1)}{f^{\prime}(1)}\right) \\
& \leqslant c f^{\prime \prime}(1) I\left\{f^{\prime}(1) \geqslant 1\right\} .
\end{aligned}
$$

Эта оценка показывает, что условие (3) влечет (9). В частности, отсюда следует, что условие $\mathscr{A}$ слабее, чем совокупность ограничений (2)-(3). 
3 а м е ч а н и е 2. Предположение об абсолютной непрерывности распределения $X$ можно ослабить. Однако это требует более деликатных (и временами утомительных) рассуждений, связанных с вводимыми позднее функциями восстановления и гармоническими функциями, и мы не будем этого делать.

3 а м е ч а и е 3 . Если $\eta_{1} \leqslant C<\infty$ с вероятностью 1 и выполнено (5), то условие (6) выполнено автоматически.

Существуют два основных подхода к нахождению асимптотики вероятности невырождения в критических и докритических ВПСС. Первый из них, предложенный М.В. Козловым в [10], опирается на свойства нижних лестничных моментов сопровождающего случайного блуждания ВПСС. При втором подходе, принадлежащем И. Гейгеру и Г. Керстингу [6], ключевым моментом является переход (при помощи преобразования «крамеровского» типа) от исходного однородного ветвящегося процесса в случайной среде к некоторому неоднородному процессу с «приятными» свойствами. Метод, при помощи которого будут доказаны соотношения (7) и (8), является развитием идей работ [6] и [7] и использует как переход от изучения исходного ветвящегося процесса в случайной среде к исследованию некоторого неоднородного процесса, так и тот факт, что для преобразованного процесса точка последнего достижения максимума сопровождающего случайного блуждания на отрезке $[0, n]$ разбивает на (условно) независимые отрезки не только траекторию этого случайного блуждания, но и случайную последовательность производящих функций, породившую эту траекторию.

2. Преобразование мер, связанных с процессом $Z_{n}$. Пусть $\Pi$ - множество всех бесконечномерных векторов (1), а $\Sigma$ - естественная $\sigma$-алгебра, порожденная подмножествами П. Согласно нашему определению, производящую функцию $f_{n}(s)$ числа непосредственных потомков частиц $n$-го поколения можно рассматривать как случайный вектор $\pi_{n}$ на вероятностном пространстве $(\Pi, \Sigma, \mathbf{P})$, в то время как совместное распределение набора $f_{0}(s), f_{1}(s), \ldots, f_{n-1}(s)$ задается естественной продакт-мерой $\mathbf{P}^{n}=\mathbf{P} \times \mathbf{P} \times \cdots \times \mathbf{P}$ на измеримом пространстве

$$
\left(\Pi^{n}, \Sigma^{n}\right)=(\Pi, \Sigma) \times(\Pi, \Sigma) \times \cdots \times(\Pi, \Sigma)
$$

а вся последовательность $f_{0}(s), f_{1}(s), \ldots, f_{n-1}(s), \ldots$ определена на измеримом пространстве $\left(\Pi^{\infty}, \Sigma^{\infty}\right)$ и распределена в соответствии с мерой $\mathbf{P}^{\infty}$ (по теореме Ионеску Тулчи о продолжении меры и существовании случайной последовательности (см., например, [14], гл. II, §9) такая вероятностная мера существует и единственна). Ясно, что в этом случае величины

$$
X_{n+1}:=\ln \left(\sum_{i=0}^{\infty} i \pi_{n}^{(i)}\right)=\ln f_{n}^{\prime}(1), \quad n \in \mathbf{N}_{0}
$$


и $S_{0}=0, S_{n}=X_{1}+\cdots+X_{n}$ будут корректно определенными случайными величинами на $\left(\Pi^{\infty}, \Sigma^{\infty}\right)$ относительно меры $\mathbf{P}^{\infty}$, если мы предположим (а далее мы будем рассматривать только такой случай), что $\mathbf{P}\left\{\pi_{n}^{(0)}=1\right\}=0$.

В последующем изложении нам будет удобно перейти от первоначальной меры $\mathbf{P}^{\infty}$ на $\Sigma^{\infty}$ к мере $\widehat{\mathbf{P}}^{\infty}$ на $\Sigma^{\infty}$, положив для любой измеримой ограниченной функции $\psi_{n}\left(y_{1}, \ldots, y_{n}\right), y_{j} \in \Pi, n=1,2, \ldots$,

$$
\widehat{\mathbf{E}}^{\infty}\left[\psi\left(f_{0}, f_{1}, \ldots, f_{n-1}\right)\right]=\lambda^{-n} \mathbf{E}\left[e^{S_{n}} \psi\left(f_{0}, f_{1}, \ldots, f_{n-1}\right)\right],
$$

где $\lambda=\mathbf{E} f_{0}^{\prime}(1)=\mathbf{E} Z_{1}$. В частности, для любого набора измеримых множеств $A_{0}, A_{1}, \ldots, A_{n-1} \subseteq \Sigma$

$$
\begin{aligned}
& \widehat{\mathbf{P}}^{\infty}\left\{f_{0} \in A_{0}, f_{1} \in A_{1}, \ldots, f_{n-1} \in A_{n-1}\right\} \\
& \quad=\lambda^{-n} \mathbf{E}\left[e^{S_{n}} I\left(f_{0} \in A_{0}, f_{1} \in A_{1}, \ldots, f_{n-1} \in A_{n-1}\right)\right]
\end{aligned}
$$

и, как следствие,

$$
\widehat{\mathbf{E}}^{\infty}\left[\ln f_{0}^{\prime}(1)\right]=\lambda^{-1} \mathbf{E}\left[e^{X_{1}} \ln f_{0}^{\prime}(1)\right]=\lambda^{-1} \mathbf{E}\left[f_{0}^{\prime}(1) \ln f_{0}^{\prime}(1)\right]=0,
$$

если начальный процесс был промежуточно докритическим. Обращаясь еще раз к теореме Ионеску Тулчи, мы видим, что такая вероятностная мера существует и единственна. Далее, для упрощения обозначений, будем писать $\widehat{\mathbf{P}}$ вместо $\widehat{\mathbf{P}}^{\infty}$ и $\widehat{\mathbf{E}}$ вместо $\widehat{\mathbf{E}}^{\infty}$.

Очевидно, что

$$
\mathbf{E}\left[\psi\left(f_{0}, f_{1}, \ldots, f_{n-1}\right)\right]=\lambda^{n} \widehat{\mathbf{E}}\left[e^{-S_{n}} \psi\left(f_{0}, f_{1}, \ldots, f_{n-1}\right)\right] .
$$

Отсюда, полагая

$$
\begin{aligned}
& f_{k, n}(s)=f_{k}\left(f_{k+1}\left(\cdots\left(f_{n-1}(s)\right) \cdots\right)\right), \quad 0 \leqslant k \leqslant n-1, \\
& f_{n, n}(s)=1, \quad f_{n, 0}(s)=f_{n-1}\left(f_{n-2}\left(\cdots\left(f_{0}(s)\right) \cdots\right)\right), \quad n \geqslant 1,
\end{aligned}
$$

и замечая, что в силу симметрии

$$
\mathbf{P}\left\{Z_{n}>0\right\}=\mathbf{E}\left(1-f_{0, n}(0)\right)=\mathbf{E}\left(1-f_{n, 0}(0)\right),
$$

получаем

$$
\mathbf{P}\left\{Z_{n}>0\right\}=\lambda^{n} \widehat{\mathbf{E}}\left[e^{-S_{n}}\left(1-f_{n, 0}(0)\right)\right] .
$$

Кроме того, если условие (2) нарушено, то, согласно (5), для $\alpha \in(1,2]$

$$
\widehat{\mathbf{P}}\{X>t\}=\lambda^{-1} \mathbf{E} e^{X} I\{X>t\} \sim \frac{L(t)}{\lambda t^{\alpha}}, \quad t \rightarrow \infty .
$$

Замечая далее, что

$$
\widehat{\mathbf{P}}\{X<-t\}=\lambda^{-1} \mathbf{E} e^{X} I\{X<-t\} \leqslant \lambda^{-1} e^{-t},
$$

мы видим, что при $\alpha \in(1,2)$ распределение случайной величины $X$ (относительно меры $\widehat{\mathbf{P}}$ ) принадлежит к области притяжения спектрально положительного закона с характеристической функцией

$$
\chi(t)=\exp \left\{-c|t|^{\alpha}\left(1+i \frac{t}{|t|} \operatorname{tg} \frac{\pi \alpha}{2}\right)\right\} .
$$


Представления (12) и (13) играют существенную роль в последующем анализе. Однако прежде чем использовать их, нам необходимо доказать ряд вспомогательных утверждений.

3. Свойства случайных блужданий и ветвящихся процессов относительно меры $\widehat{\mathbf{P}}$. С этого момента будем предполагать, если не оговорено иное, что набор $X, X_{1}, X_{2}, \ldots$ состоит из независимых случайных величин с общей функцией распределения, подчиняюшейся условию (A1).

Положим

$$
\tau=\min \left\{k \geqslant 1: S_{k}<0\right\}, \quad \tau^{0}=\min \left\{k \geqslant 1: S_{k} \geqslant 0\right\} .
$$

В последующем символами $c, c_{1}, \ldots$ будем обозначать положительные константы, а символами $L_{1}(n), L_{2}(n), \ldots$ - функции, медленно меняющиеся при $n \rightarrow \infty$. Они могут быть различными в разных формулах.

Следующая лемма является переформулировкой леммы 5 paботы [15].

Лемма 1 (см. [15]). Если выполнено условие (A1), то для любого $t>0$ существует медленно меняющаяся функиия $L_{2}(n)$ такая, что

$$
\widehat{\mathbf{E}}\left[e^{-t S_{n}} ; \tau>n\right]=O\left(n^{-(1 / \alpha+1)} L_{2}(n)\right), \quad n \rightarrow \infty .
$$

Обозначим $0=\Gamma_{0}<\Gamma_{1}<\Gamma_{2}<\cdots$ строгие возрастающие лестничные моменты случайного блуждания $\left\{S_{n}\right\}_{n \geqslant 0}$ :

$$
\Gamma_{j+1}:=\min \left(n>\Gamma_{j}: S_{n}>S_{\Gamma_{j}}\right), \quad j \geqslant 0 .
$$

Положим

$$
\mu_{n}:=\max _{0 \leqslant k \leqslant n} S_{k}, \quad m_{n}(x)=\widehat{\mathbf{P}}\left\{\mu_{n} \leqslant x\right\}, \quad x \geqslant 0 .
$$

Обозначим $U(x)=0, x<0, U(0)=1$,

$$
U(x)=\sum_{k=0}^{\infty} \widehat{\mathbf{P}}\left\{S_{k} \leqslant x, \tau>k\right\}=\sum_{j=0}^{\infty} \widehat{\mathbf{P}}\left\{S_{\Gamma_{j}} \leqslant x\right\}, \quad x>0 .
$$

Из леммы 1 работы [9] вытекает, что при наших условиях $U(x)$ является гармонической функцией, т.е., для любого $x \geqslant 0$

$$
\widehat{\mathbf{E}} U\left(x-X_{1}\right)=U(x) \text {. }
$$

Положим

$$
\rho=\frac{1}{2}-\frac{1}{\pi \alpha} \operatorname{arctg}\left(-\operatorname{tg} \frac{\pi \alpha}{2}\right)=1-\frac{1}{\alpha} .
$$

Две леммы, формулируемые ниже, являются следствиями лемм 7 и 9 статьи [15].

Лемма 2 (см. [15]). Если выполнено условие (A1), то существует медленно меняющаяся функиия $L_{3}(x)$ такая, что

$$
U(x) \backsim x^{\alpha(1-\rho)} L_{3}(x)=x L_{3}(x), \quad x \rightarrow \infty .
$$


Лемма 3 (см. [15]). Если выполнено условие (A1), то существует медленно меняюшаяся функция $L_{4}(x)$ такая, ито при $n \rightarrow \infty$

$$
\begin{gathered}
m_{n}(x) \backsim U(x) n^{-1 / \alpha} L_{4}(n) \quad \text { для любого фиксированного } x>0, \\
\widehat{\mathbf{P}}\left\{\tau^{0}>n\right\}=m_{n}(0) \sim n^{-1 / \alpha} L_{4}(n),
\end{gathered}
$$

u найдется константа $c_{1}>0$ такая, что для всех $x>0$

$$
m_{n}(x) \leqslant c_{1} U(x) n^{-1 / \alpha} L_{4}(n) .
$$

Формула, указанная в следующей лемме, играет существенную роль в наших рассуждениях. Она получена путем естественных преобразований из соответствующей формулы работы [6].

Лемма 4 (сравните с [6]). Пусть $f_{j} \neq 1,0 \leqslant j \leqslant n-1$. Для любого $0 \leqslant s<1$

$$
\frac{e^{S_{n}}}{1-f_{n, 0}(s)}=\frac{1}{1-s}+\sum_{j=1}^{n} \eta_{j, 0}(s) e^{S_{j}}
$$

2de

$$
\eta_{j, 0}(s)=\frac{1}{1-f_{j, 0}(s)}-\frac{1}{f_{j-1}^{\prime}(1)\left(1-f_{j-1,0}(s)\right)} \leqslant \eta_{j}:=\frac{f_{j-1}^{\prime \prime}(1)}{\left(f_{j-1}^{\prime}(1)\right)^{2}} .
$$

Для того, чтобы идти дальше, нам необходимо ввести еще одну вероятностную меру.

Для любого ограниченного функционала $\psi$, который зависит только от $f_{0}, f_{1}, \ldots, f_{k-1}$, положим

$$
\widehat{\mathbf{E}}^{-}\left[\psi\left(f_{0}, \ldots, f_{k-1}\right)\right]:=\widehat{\mathbf{E}}\left[\psi\left(f_{0}, \ldots, f_{k-1}\right) U\left(-S_{k}\right) I\left\{\tau^{0}>k\right\}\right] .
$$

Заметим, что ввиду (17) это определение корректно. В частности, мы получаем вероятностную меру

$$
\widehat{\mathbf{P}}^{-}\left\{f_{j} \in d x_{j}, j=0,1, \ldots, k-1\right\}=\widehat{\mathbf{E}}^{-} I\left\{f_{j} \in d x_{j}, j=0,1, \ldots, k-1\right\} .
$$

Лемма 5. Если выполнено условие (A1), то для любого ограниченного функиионала $\psi$ и $k \geqslant 1$ справедливо представление

$$
\widehat{\mathbf{E}}^{-}\left[\psi\left(f_{0}, \ldots, f_{k-1}\right)\right]=\lim _{n \rightarrow \infty} \widehat{\mathbf{E}}\left[\psi\left(f_{0}, \ldots, f_{k-1}\right) \mid \tau^{0}>n\right] .
$$

Д о к а з а т е л в с т в о. Так как производящие функции $f_{0}, \ldots$, $f_{k-1}$ не зависят от случайной последовательности $\left\{S_{m}-S_{k}, m=k, k+\right.$ $1, \ldots, n\}$, то

$$
\widehat{\mathbf{E}}\left[\psi\left(f_{0}, \ldots, f_{k-1}\right) \mid \tau^{0}>n\right]=\frac{\widehat{\mathbf{E}}\left[\psi\left(f_{0}, \ldots, f_{k-1}\right) m_{n-k}\left(-S_{k}\right) I\left\{\tau^{0}>k\right\}\right]}{m_{n}(0)} .
$$

Вспоминая соотношения (20)-(21) и применяя теорему об ограниченной сходимости, получаем (26).

Доказательство закончено. 
Положим $\xi_{n}(s)=e^{-S_{n}}\left(1-f_{n, 0}(s)\right), n=1,2, \ldots$

Лемма 6. Если выполнено условие $\mathscr{A}$, то для любого $s \in[0,1) u$ любого фиксированного $k=1,2, \ldots$

$$
\lim _{n \rightarrow \infty} \widehat{\mathbf{P}}\left\{\xi_{k}(s)<x \mid \tau^{0}>n\right\}=\widehat{\mathbf{P}}^{-}\left\{\xi_{k}(s)<x\right\} .
$$

Кроме того, на измеримом пространстве $\left(\Pi^{\infty}, \Sigma^{\infty}\right)$ найдется случайная величина $\xi_{\infty}(s)$ такая, что при $k \rightarrow \infty$

$$
\xi_{k}(s) \rightarrow \xi_{\infty}(s) \quad \widehat{\mathbf{P}}-\text { n.н. }
$$

причем $\widehat{\mathbf{P}}-\left\{\xi_{\infty}(s)>0\right\}=1$.

Д о к а з а т е л в с т в о. Как и в предыдущей лемме,

$$
\widehat{\mathbf{P}}\left\{\xi_{k}(s)<x \mid \tau^{0}>n\right\}=\left(m_{n}(0)\right)^{-1} \widehat{\mathbf{E}}\left[I\left\{\xi_{k}(s)<x\right\} m_{n-k}\left(-S_{k}\right) I\left\{\tau^{0}>k\right\}\right] .
$$

Применение к этому равенству теоремы об ограниченной сходимости завершает доказательство равенства (27). Для доказательства (28) заметим, что в силу того, что

$$
0 \leqslant \xi_{k}(s)=e^{-S_{k}}\left(1-f_{k, 0}(s)\right) \leqslant e^{-S_{k}} f_{k-1}^{\prime}(1)\left(1-f_{k-1,0}(s)\right)=\xi_{k-1}(s),
$$

последовательность $\xi_{k}(s)$ монотонно убывает по $k$. Следовательно, существует случайная величина $\xi_{\infty}(s)$ такая, что $\xi_{k}(s) \rightarrow \xi_{\infty}(s) \widehat{\mathbf{P}}^{-}$-п.н. Покажем, что $\widehat{\mathbf{P}}^{-}\left\{\xi_{\infty}(s)>0\right\}=1$. Действительно, ввиду леммы 4 и неравенства Маркова для любого $\varepsilon_{0} \in(0,1]$ и любого $x>0$

$$
\begin{aligned}
\widehat{\mathbf{P}}^{-}\left\{\frac{e^{S_{k}}}{1-f_{k, 0}(s)}>x\right\} & \leqslant \widehat{\mathbf{P}}^{-}\left\{\frac{1}{1-s}+\sum_{j=1}^{k} \eta_{j} e^{S_{j}}>x\right\} \\
& \leqslant x^{-\varepsilon_{0}} \widehat{\mathbf{E}}^{-}\left\{\frac{1}{(1-s)^{\varepsilon_{0}}}+\sum_{j=1}^{k} \eta_{j}^{\varepsilon_{0}} e^{\varepsilon_{0} S_{j}}\right\} .
\end{aligned}
$$

Таким образом, доказательство леммы будет завершено, если мы покажем, что

$$
\widehat{\mathbf{E}}^{-}\left[\sum_{j=1}^{\infty} \eta_{j}^{\varepsilon_{0}} e^{\varepsilon_{0} S_{j}}\right]<\infty .
$$

Для доказательства этого факта вспомним, что в силу условия $\mathscr{A}$ и относительно меры $\widehat{\mathbf{P}}$

$\left(\mathrm{A1}^{\prime}\right) X_{1}$ принадлежит области притяжения устойчивого закона с параметром $\alpha \in(1,2]$;

$\left(\mathrm{A} 2^{\prime}\right)$ при $\alpha<2$ сушествуют $\varepsilon_{0} \in(0,1]$ и $\delta_{0} \in(0,1)$ такие, что

$$
\begin{aligned}
\widehat{\mathbf{E}}[ & \left.\eta_{1}^{\varepsilon_{0}}\left(\left(1+X_{1}\right)^{\alpha-1+\delta_{0}} I\left\{X_{1} \geqslant 0\right\}+\left(1-X_{1}\right)^{\alpha-1+\delta_{0}} e^{\varepsilon_{0} X_{1}} I\left\{X_{1}<0\right\}\right)\right] \\
= & \widehat{\mathbf{E}}\left[\eta _ { 1 } ^ { \varepsilon _ { 0 } } \left(\left(1+X_{1}\right)^{\alpha(1-\rho)+\delta_{0}} I\left\{X_{1} \geqslant 0\right\}\right.\right. \\
& \left.\left.\quad+\left(1-X_{1}\right)^{\alpha(1-\rho)+\delta_{0}} e^{\varepsilon_{0} X_{1}} I\left\{X_{1}<0\right\}\right)\right] \\
= & \lambda^{-1} \mathbf{E}\left[e^{X_{1}} \eta_{1}^{\varepsilon_{0}}\left(1+X_{1}\right)^{\alpha(1-\rho)+\delta_{0}} I\left\{X_{1} \geqslant 0\right\}\right] \\
& +\lambda^{-1} \mathbf{E}\left[e^{X_{1}} \eta_{1}^{\varepsilon_{0}}\left(1-X_{1}\right)^{\alpha(1-\rho)+\delta_{0}} e^{\varepsilon_{0} X_{1}} I\left\{X_{1}<0\right\}\right]<\infty .
\end{aligned}
$$


$\left(\mathrm{A} 3^{\prime}\right)$ при $\alpha=2$ условие (30) выполнено с $\delta_{0}=0$.

Условия $\left(\mathrm{A} 1^{\prime}\right)-\left(\mathrm{A} 3^{\prime}\right)$ являются в точности условиями леммы 11 работы [15], которая утверждает, что при их выполнении справедливо неравенство (29).

Лемма доказана.

Лемма 7. Если выполнено условие $\mathscr{A}$, то для любого $s \in[0,1)$

$$
\lim _{n \rightarrow \infty} \widehat{\mathbf{P}}\left\{\xi_{n}(s)<x \mid \tau^{0}>n\right\}=\widehat{\mathbf{P}}^{-}\left\{\xi_{\infty}(s)<x\right\}
$$

во всех точках непрерывности распределения $\xi_{\infty}(s)$.

Д о к а за те ль с т в о. В силу леммы 6 для того, чтобы убедиться в справедливости (31), достаточно показать, что для любого $\varepsilon>0$

$$
\lim _{k \rightarrow \infty} \limsup _{n \rightarrow \infty} \widehat{\mathbf{P}}\left\{\xi_{k}(s)-\xi_{n}(s)>\varepsilon \mid \tau^{0}>n\right\}=0 .
$$

Для любого $\gamma>1$

$$
\begin{aligned}
& \widehat{\mathbf{P}}\left\{\xi_{k}(s)-\xi_{n}(s)>\varepsilon \mid \tau^{0}>n\right\}=\left(m_{n}(0)\right)^{-1} \widehat{\mathbf{P}}\left\{\xi_{k}(s)-\xi_{n}(s)>\varepsilon ; \tau^{0}>n\right\} \\
& \quad \leqslant\left(m_{n}(0)\right)^{-1} \widehat{\mathbf{P}}\left\{\xi_{k}(s)-\xi_{n}(s)>\varepsilon ; \tau^{0}>n \gamma\right\} \\
& \quad+\left(m_{n}(0)\right)^{-1} \widehat{\mathbf{P}}\left\{\tau^{0} \leqslant n \gamma ; \tau^{0}>n\right\} .
\end{aligned}
$$

Согласно соотношению (22)

$$
\begin{aligned}
\widehat{\mathbf{P}} & \left\{\xi_{k}(s)-\xi_{n}(s)>\varepsilon ; \tau^{0}>n \gamma\right\} \\
& =\widehat{\mathbf{E}}\left[I\left\{\xi_{k}(s)-\xi_{n}(s)>\varepsilon\right\} m_{n(\gamma-1)}\left(-S_{n}\right) I\left\{\tau^{0}>n\right\}\right] \\
& \leqslant \frac{c_{1} L_{4}(n(\gamma-1))}{(n(\gamma-1))^{1 / \alpha}} \widehat{\mathbf{E}}\left[I\left\{\xi_{k}(s)-\xi_{n}(s)>\varepsilon\right\} U\left(-S_{n}\right) I\left\{\tau^{0}>n\right\}\right] \\
& =\frac{c_{1} L_{4}(n(\gamma-1))}{(n(\gamma-1))^{1 / \alpha}} \widehat{\mathbf{P}}-\left\{\xi_{k}(s)-\xi_{n}(s)>\varepsilon\right\} .
\end{aligned}
$$

Ввиду эквивалентности $(20)$ для достаточно больших значений $n$

$$
\begin{aligned}
& \left(m_{n}(0)\right)^{-1} \widehat{\mathbf{P}}\left\{\xi_{k}(s)-\xi_{n}(s)>\varepsilon ; \tau^{0}>n \gamma\right\} \\
& \quad \leqslant c_{3} \frac{n^{1 / \alpha} L_{4}(n(\gamma-1))}{(n(\gamma-1))^{1 / \alpha} L_{4}(n)} \widehat{\mathbf{P}}^{-}\left(\xi_{k}(s)-\xi_{n}(s)>\varepsilon\right) .
\end{aligned}
$$

Отсюда, в силу свойств медленно меняющихся функций и сходимости в (28), выводим, что

$$
\begin{gathered}
\lim _{k \rightarrow \infty} \limsup _{n \rightarrow \infty}\left(m_{n}(0)\right)^{-1} \widehat{\mathbf{P}}\left\{\xi_{k}(s)-\xi_{n}(s)>\varepsilon ; \tau^{0}>n \gamma\right\} \\
\leqslant c_{3}(\gamma-1)^{-1 / \alpha} \lim _{k \rightarrow \infty} \widehat{\mathbf{P}}^{-}\left\{\xi_{k}(s)-\xi_{\infty}(s)>\varepsilon\right\}=0 .
\end{gathered}
$$

С другой стороны, снова в силу (20)

$$
\begin{aligned}
\lim _{\gamma \downarrow 1} \lim _{n \rightarrow \infty}\left(m_{n}(0)\right)^{-1} \widehat{\mathbf{P}}\left\{\tau^{0} \leqslant n \gamma ; \tau^{0}>n\right\} & =1-\lim _{\gamma \downarrow 1} \lim _{n \rightarrow \infty} \frac{m_{n \gamma}(0)}{m_{n}(0)} \\
& =1-\lim _{\gamma \downarrow 1} \gamma^{-1 / \alpha}=0 .
\end{aligned}
$$


Комбинируя оценки (33)-(35), мы получаем равенство (32), что и доказывает лемму.

4. Доказательство предельной теоремы. Теперь у нас все готово для доказательства нашей предельной теоремы.

Сначала докажем представление (4). Пусть

$$
T(n)=\max \left\{k \in[0, n]: S_{j} \leqslant S_{k}, j \leqslant k, \text { и } S_{j}<S_{k}, n \geqslant j>k\right\}
$$

обозначает последний момент достижения максимума последовательностью $S_{0}, S_{1}, \ldots, S_{n}$. В силу (12) и формулы полной вероятности

$$
\lambda^{-n} \mathbf{P}\left\{Z_{n}>0\right\}=\sum_{k=0}^{n} \widehat{\mathbf{E}}\left[e^{-S_{n}}\left(1-f_{n, 0}(0)\right) ; T(n)=k\right] .
$$

Заметим, что

$$
1-f_{n, 0}(0) \leqslant f_{n-1}^{\prime}(1)\left(1-f_{n-1,0}(0)\right)=e^{X_{n}}\left(1-f_{n-1,0}(0)\right) \leqslant e^{\min _{0 \leqslant j \leqslant n}\left(S_{n}-S_{j}\right)} .
$$

Следовательно,

$$
\begin{aligned}
& \widehat{\mathbf{E}}\left[e^{-S_{n}}\left(1-f_{n, 0}(0)\right) ; T(n)=k\right] \leqslant \widehat{\mathbf{E}}\left[e^{-S_{n}} e^{\min _{0 \leqslant j \leqslant n}\left(S_{n}-S_{j}\right)} ; T(n)=k\right] \\
& \quad=\widehat{\mathbf{E}}\left[e^{-\max _{0 \leqslant j \leqslant n} S_{j}} ; T(n)=k\right]=\widehat{\mathbf{E}}\left[e^{-S_{k}} ; T(n)=k\right] .
\end{aligned}
$$

Далее, согласно принципу двойственности

$$
\begin{aligned}
\widehat{\mathbf{E}}\left[e^{-S_{k}} ; T(n)=k\right] & \left.=\widehat{\mathbf{E}} \mid e^{-S_{k}} ; S_{j} \leqslant S_{k}, j \leqslant k, \text { и } S_{j}<S_{k}, n \geqslant j>k\right] \\
& =\widehat{\mathbf{E}}\left[e^{-S_{k}} ; \tau>k\right] \widehat{\mathbf{P}}\left\{\tau^{0}>n-k\right\} .
\end{aligned}
$$

Ввиду леммы 1 и оценки (21)

$\widehat{\mathbf{E}}\left[e^{-S_{k}} ; \tau>k\right] \widehat{\mathbf{P}}\left\{\tau^{0}>n-k\right\} \leqslant c k^{-1 / \alpha-1} L_{2}(k)(n-k+1)^{-1 / \alpha} L_{4}(n-k+1)$.

Следовательно, для фиксированных $N$ и $\varepsilon \in(0,1)$

$$
\begin{aligned}
& \sum_{k=N+1}^{n} \widehat{\mathbf{E}}\left[e^{-S_{k}} ; T(n)=k\right] \\
& \leqslant c_{1} \sum_{k=N+1}^{n(1-\varepsilon)} k^{-(1 / \alpha+1)} L_{2}(k)(n-k+1)^{-1 / \alpha} L_{4}(n-k+1) \\
& \quad+c_{2} \sum_{k=n(1-\varepsilon)}^{n} k^{-(1 / \alpha+1)} L_{2}(k)(n-k+1)^{-1 / \alpha} L_{4}(n-k+1) \\
& \leqslant c_{3} n^{-1 / \alpha} L_{4}(n) \sum_{k=N+1}^{\infty} k^{-(1 / \alpha+1)} L_{2}(k) \\
& \quad+c_{4} n^{-(1 / \alpha+1)} L_{2}(n) \sum_{k=0}^{n \varepsilon}(k+1)^{-1 / \alpha} L_{4}(k+1) .
\end{aligned}
$$

Ясно, что для любого $\delta>0$ найдутся $N_{0}$ и $n_{0}$ такие, что для всех $N \geqslant N_{0}$

$$
\sum_{k=N+1}^{\infty} k^{-(1 / \alpha+1)} L_{2}(k) \leqslant \delta
$$


а для всех $n \geqslant n_{0}$

$$
\begin{aligned}
n^{-(1 / \alpha+1)} L_{2}(n) \sum_{k=0}^{n \varepsilon}(k+1)^{-1 / \alpha} L_{4}(k+1) & \leqslant c n^{-(1 / \alpha+1)} L_{2}(n) n^{1-1 / \alpha} L_{4}(n) \\
& \leqslant \delta n^{-1 / \alpha} L_{4}(n) .
\end{aligned}
$$

Отсюда при $n \geqslant n_{0}$ и $n+1 \geqslant N \geqslant N_{0}$

$$
\sum_{k=N+1}^{n} \widehat{\mathbf{E}}\left[e^{-S_{k}} ; T(n)=k\right] \leqslant \delta c n^{-1 / \alpha} L_{4}(n)
$$

или

$$
\lambda^{-n} \mathbf{P}\left\{Z_{n}>0\right\}=\sum_{k=0}^{N} \widehat{\mathbf{E}}\left[e^{-S_{n}}\left(1-f_{n, 0}(0)\right) ; T(n)=k\right]+\delta_{n, N} n^{-1 / \alpha} L_{4}(n),
$$

где $\lim _{N \rightarrow \infty} \lim _{n \rightarrow \infty} \delta_{n, N}=0$. С другой стороны, нетрудно проверить, что

$$
\begin{aligned}
& \widehat{\mathbf{E}}\left[e^{-S_{n}}\left(1-f_{n, 0}(0)\right) ; T(n)=k\right] \\
& =\widehat{\mathbf{E}}\left[e^{-S_{k}-\left(S_{n}-S_{k}\right)}\left(1-f_{n, k}\left(f_{k, 0}(0)\right)\right) ; T(n)=k\right] \\
& =\int_{0}^{\infty} \int_{0}^{1} e^{-x} \widehat{\mathbf{E}}\left[e^{-S_{n-k}^{*}}\left(1-F_{n-k, 0}(y)\right) ;\right. \\
& \left.\tau_{*}^{0}>n-k\right] d \widehat{\mathbf{P}}\left\{S_{k} \leqslant x, f_{k, 0}(0) \leqslant y ; \tau>k\right\},
\end{aligned}
$$

где $S_{n-k}^{*}$ и $\tau_{*}^{0}$ - случайные величины, аналогичные $S_{n-k}$ и $\tau^{0}$, но построенные при помощи последовательности $F_{j}(y) \stackrel{d}{=} f_{j+k}(y), j=0,1, \ldots, n-$ $k-1,0 \leqslant y \leqslant 1$; причем $F_{n-k, 0}(y)=F_{n-k-1}\left(F_{n-k-2}\left(\cdots\left(F_{0}(y)\right) \cdots\right)\right.$. В силу леммы 7 и теоремы об ограниченной сходимости при любом фиксированном $y$

$$
\lim _{n \rightarrow \infty} \widehat{\mathbf{E}}\left[e^{-S_{n-k}^{*}}\left(1-F_{n-k, 0}(y)\right) \mid \tau_{*}^{0}>n-k\right]=\widehat{\mathbf{E}}^{-}\left[\xi_{\infty}(y)\right]>0 .
$$

Отсюда, используя еще раз теорему об ограниченной сходимости и тот факт, что при любом фиксированном $k$ и $n \rightarrow \infty$

$$
\widehat{\mathbf{P}}\left\{\tau_{*}^{0}>n-k\right\}=\widehat{\mathbf{P}}\left\{\tau^{0}>n-k\right\} \sim(n-k)^{-1 / \alpha} L_{4}(n-k) \sim n^{-1 / \alpha} L_{4}(n),
$$

заключаем, что

$$
\begin{aligned}
& \lim _{n \rightarrow \infty} n^{1 / \alpha}\left(L_{4}(n)\right)^{-1} \widehat{\mathbf{E}}\left[e^{-S_{n}}\left(1-f_{n, 0}(0)\right) ; T(n)=k\right] \\
& \quad=\int_{0}^{\infty} \int_{0}^{1} e^{-x} \widehat{\mathbf{E}}^{-}\left[\xi_{\infty}(y)\right] d \widehat{\mathbf{P}}\left\{S_{k} \leqslant x, f_{k, 0}(0) \leqslant y ; \tau>k\right\} .
\end{aligned}
$$

Следовательно,

$$
\begin{aligned}
\lim _{n \rightarrow \infty} n^{1 / \alpha}\left(L_{4}(n)\right)^{-1} \lambda^{-n} \mathbf{P}\left\{Z_{n}>0\right\} \\
\quad=\sum_{k=0}^{\infty} \int_{0}^{\infty} \int_{0}^{1} e^{-x} \widehat{\mathbf{E}}^{-}\left[\xi_{\infty}(y)\right] d \widehat{\mathbf{P}}\left\{S_{k} \leqslant x, f_{k, 0}(0) \leqslant y ; \tau>k\right\} \\
\quad=\sum_{k=0}^{\infty} \widehat{\mathbf{E}}^{-}\left[e^{-S_{k}} \xi_{\infty}\left(f_{k, 0}(0)\right) I\{\tau>k\}\right] \in(0, \infty) .
\end{aligned}
$$


Таким образом, соотношение (4) доказано. Заметим, что случайные величины $\xi_{\infty}(y)$ и $f_{k, 0}(0)$ независимы.

При помощи таких же рассуждений можно показать, что для каждого $s \in[0,1)$

$$
\begin{aligned}
\lim _{n \rightarrow \infty} n^{1 / \alpha}\left(L_{4}(n)\right)^{-1} \widehat{\mathbf{E}}\left[1-s^{Z_{n}}\right] & =\lim _{n \rightarrow \infty} n^{1 / \alpha}\left(L_{4}(n)\right)^{-1} \widehat{\mathbf{E}}\left[e^{-S_{n}}\left(1-f_{n, 0}(s)\right)\right] \\
& =\sum_{k=0}^{\infty} \widehat{\mathbf{E}}^{-}\left[e^{-S_{k}} \xi_{\infty}\left(f_{k, 0}(s)\right) I\{\tau>k\}\right] \in(0, \infty) .
\end{aligned}
$$

Отсюда

$$
\lim _{n \rightarrow \infty} \mathbf{E}\left[s^{Z_{n}} \mid Z_{n}>0\right]=1-\frac{\sum_{k=0}^{\infty} \widehat{\mathbf{E}}^{-}\left[e^{-S_{k}} \xi_{\infty}\left(f_{k, 0}(s)\right) I\{\tau>k\}\right]}{\sum_{k=0}^{\infty} \widehat{\mathbf{E}}^{-}\left[e^{-S_{k}} \xi_{\infty}\left(f_{k, 0}(0)\right) I\{\tau>k\}\right]} .
$$

Правая часть (37) и есть производяшая функция $\phi(s)$, упомянутая в формуле (8) при формулировке теоремы 1.

Покажем, что предельная случайная величина, определяемая производящей функцией, стояшей в правой части (37), является собственной.

Для $x>0$ и $n \geqslant 1$ положим

$$
J_{n, x}\left(f_{0}, \ldots, f_{n-1}\right):=\mathbf{P}\left\{Z_{n} \geqslant x \mid f_{0}, \ldots, f_{n-1}\right\} .
$$

В силу того, что случайные функции $f_{j}$ перестановочны,

$$
\begin{aligned}
\mathbf{P}\left\{Z_{n} \geqslant x\right\}= & \mathbf{E}\left[J_{n, x}\left(f_{n-1}, \ldots, f_{0}\right)\right] \\
\leqslant & \mathbf{E}\left[J_{n, x}\left(f_{n-1}, \ldots, f_{0}\right) ; \mu_{n}>x^{1 / 2}\right] \\
& +\mathbf{E}\left[J_{n, x}\left(f_{n-1}, \ldots, f_{0}\right) ; \mu_{n} \leqslant x^{1 / 2}\right],
\end{aligned}
$$

где, как и раньше, $\mu_{n}=\max _{0 \leqslant k \leqslant n} S_{k}$. Ясно, что

$$
\begin{array}{rl}
\lambda^{-n} & \mathbf{E}\left[J_{n, x}\left(f_{n-1}, \ldots, f_{0}\right) ; \mu_{n}>x^{1 / 2}\right] \\
& =\widehat{\mathbf{E}}\left[e^{-S_{n}} J_{n, x}\left(f_{n-1}, \ldots, f_{0}\right) ; \mu_{n}>x^{1 / 2}\right] \\
& \leqslant \widehat{\mathbf{E}}\left[e^{-S_{n}}\left(1-f_{n, 0}(0)\right) ; \mu_{n}>x^{1 / 2}\right] \\
& \leqslant \widehat{\mathbf{E}}\left[e^{-\mu_{n}} ; \mu_{n}>x^{1 / 2}\right] \leqslant \sum_{k \geqslant\left[x^{1 / 2}\right]} e^{-k} \widehat{\mathbf{P}}\left\{k<\mu_{n} \leqslant k+1\right\} \\
& \leqslant \sum_{k \geqslant\left[x^{1 / 2}\right]} e^{-k} m_{n}(k+1) \leqslant c_{1} n^{-1 / \alpha} L_{4}(n) \sum_{k \geqslant\left[x^{1 / 2}\right]} e^{-k} U(k+1),
\end{array}
$$

где на последнем шаге было использовано (22). Ввиду (19) мы видим, что

$$
\limsup _{x \rightarrow \infty} \limsup _{n \rightarrow \infty} \lambda^{-n} n^{1 / \alpha} L_{4}(n) \mathbf{E}\left[J_{n, x}\left(f_{n-1}, \ldots, f_{0}\right) ; \mu_{n}>x^{1 / 2}\right]=0 .
$$

Далее, в силу неравенства Чебышева и соотношения (22)

$$
\begin{aligned}
& \lambda^{-n} \mathbf{E}\left[J_{n, x}\left(f_{n-1}, \ldots, f_{0}\right) ; \mu_{n} \leqslant x^{1 / 2}\right] \leqslant x^{-1} \lambda^{-n} \mathbf{E}\left[e^{S_{n}} ; \mu_{n} \leqslant x^{1 / 2}\right] \\
& \quad=x^{-1} \widehat{\mathbf{P}}\left\{\mu_{n} \leqslant x^{1 / 2}\right\} \leqslant c_{1} n^{-1 / \alpha} L_{4}(n) x^{-1} U\left(x^{1 / 2}\right) .
\end{aligned}
$$




\section{Отсюда и из (19) вытекает, что}

$\limsup \limsup \lambda^{-n} n^{1 / \alpha}\left(L_{4}(n)\right)^{-1} \mathbf{E}\left[J_{n, x}\left(f_{n-1}, \ldots, f_{0}\right) ; \mu_{n} \leqslant x^{1 / 2}\right]=0$.

Комбинируя (38)-(40) с (7), мы видим, что

$$
\begin{gathered}
\lim _{x \rightarrow \infty} \limsup _{n \rightarrow \infty} \mathbf{P}\left\{Z_{n}>x \mid Z_{n}>0\right\}=\lim _{x \rightarrow \infty} \limsup _{n \rightarrow \infty} \frac{\mathbf{P}\left\{Z_{n}>x\right\}}{\mathbf{P}\left\{Z_{n}>0\right\}} \\
\leqslant c \lim _{x \rightarrow \infty} \limsup _{n \rightarrow \infty} \lambda^{-n} n^{1 / \alpha}\left(L_{4}(n)\right)^{-1} \mathbf{P}\left\{Z_{n}>x\right\}=0 .
\end{gathered}
$$

Это соотношение и показывает, что случайная величина, задаваемая правой частью (37), является собственной.

Теорема доказана.

\section{СПИСОК ЛИТЕРАТУРЫ}

1. Афанасьев В. И. Предельные теоремы для умеренно докритического ветвяшегося процесса в случайной среде. - Дискретн. матем., 1998, т. 10, № 1, с. 141-157.

2. Афанасьев В.И. Предельные теоремы для промежуточно докритического и строго докритического ветвяшихся процессов в случайной среде. - Дискретн. матем., 2001, т. 13, № 1, с. 132-157.

3. Dekking $M$. On the survival probability of a branching process in a finite state i.i.d. environment. - Stoch. Proc. Appl., 1988, v. 27, № 1, p. 151-157.

4. Феллер В. Введение в теорию вероятностей и ее приложения. Т. 2. М.: Мир, 1984, $752 \mathrm{c}$.

5. Fleischmann K., Vatutin V.A. Reduced subcritical branching processes in random environment. - Adv. Appl. Probab., 1999, v. 31, № 1, p. 88-111.

6. Geiger J., Kersting G. The survival probability of a critical branching process in random environment. - Теория вероятн. и ее примен., 2000, т. 45, в. 3, с. 607-615.

7. Geiger J., Kersting G., Vatutin V.A. The survival probability of a subcritical branching process in random environment. - Ann. Inst. Henri-Poincaré (B) Probability and Statistics, 2003, v. 39, № 4, p. 593-620.

8. Guivarc'h $Y$., Liu $Q$. Propriétés asymptotiques des processus de branchement en environment alétoire. - C. R. Acad. Sci. Paris, Série I, 2001, v. 332, № 4, p. 339-344.

9. Hirano $K$. Determination of the limiting coefficient for exponential functionals of random walks with positive drift. - J. Math. Sci., Tokyo, 1998, v. 5, № 22, p. 299332.

10. Козлов М. В. Критические ветвяшиеся процессы в случайной среде. - Теория вероятн. и ее примен., 1976, т. XXI, в. 2, с. 813-825.

11. Liu $Q$. On the survival probability of a branching process in a random environment. Ann. Inst. Henri-Poincaré, 1996, v. 32, № 1, p. 1-10.

12. Нагаев $A . B$. Крамеровские большие уклонения в случае, когда сопряженное распределение имеет тяжелый хвост. - Теория вероятн. и ее примен., 1998, т. 43, B. 3 , c. $456-475$.

13. D'Souza J. C., Hambly B. M. On the survival probability of a branching process in a random environment. - Adv. Appl. Probab., 1997, v. 29, № 1, p. 38-55.

14. Ширяев A. Н. Вероятность. М.: Наука, 1980, 576 с.

15. Ватутин B. A., Дьяконова $E$. E. Ветвящиеся процессы Гальтона-Ватсона в случайной среде. I: Предельные теоремы. - Теория вероятн. и ее примен., 2002, т. 48 , в. 2 , с. $274-300$.

16. Vatutin V. A., Zubkov A. M. Branching Processes II. - J. Soviet Math., 1993, v. 67, № 6 , p. $3407-3485$. 